\title{
Volumetric feedback ablation of uterine fibroids using magnetic resonance-guided high intensity focused ultrasound therapy
}

\author{
M. J. Voogt • H. Trillaud • Y. S. Kim • W. P. Th. M. Mali • J. Barkhausen • \\ L. W. Bartels • R. Deckers • N. Frulio • H. Rhim • H. K. Lim • T. Eckey • \\ H. J. Nieminen • C. Mougenot • B. Keserci • J. Soini • T. Vaara • M. O. Köhler • \\ S. Sokka • Maurice A. A. J. van den Bosch
}

Received: 12 March 2011 /Revised: 18 August 2011 / Accepted: 29 August 2011 / Published online: 8 September 2011

(C) The Author(s) 2011. This article is published with open access at Springerlink.com

\begin{abstract}
Objective The purpose of this prospective multicenter study was to assess the safety and technical feasibility of volumetric Magnetic Resonance-guided High Intensity Focused Ultrasound (MR-HIFU) ablation for treatment of patients with symptomatic uterine fibroids.

Methods Thirty-three patients with 36 fibroids were treated with volumetric MR-HIFU ablation. Treatment capability and technical feasibility were assessed by comparison of the Non-Perfused Volumes (NPVs) with MR thermal dose predicted treatment volumes. Safety was determined by evaluation of complications or adverse events and unintended lesions. Secondary endpoints were pain and discomfort scores, recovery time and length of hospital stay. Results The mean NPV calculated as a percentage of the total fibroid volume was $21.7 \%$. Correlation
\end{abstract}

\footnotetext{
M. J. Voogt • W. P. T. M. Mali • M. A. A. J. van den Bosch ( $\varangle)$ Department of Radiology, University Medical Center Utrecht, Heidelberglaan 100,

3584 GX Utrecht, The Netherlands

e-mail: mbosch@umcutrecht.nl

H. Trillaud $\cdot$ N. Frulio

Department of Radiology, Hospital St. Andre,

Bordeaux, France

Y. S. Kim $\cdot$ H. Rhim $\cdot$ H. K. Lim

Department of Radiology, Samsung Medical Center,

Sungkyunkwan University School of Medicine,

Seoul, Republic of Korea

J. Barkhausen · T. Eckey

Department of Radiology,

University Hospital Schleswig-Holstein,

Lübeck, Germany
}

between the predicted treatment volumes and NPVs was found to be very strong, with a correlation coefficient $r$ of 0.87 . All patients tolerated the treatment well and were treated on an outpatient basis. No serious adverse events were reported and recovery time to normal activities was $2.3 \pm 1.8$ days.

Conclusion This prospective multicenter study proved that volumetric MR-HIFU is safe and technically feasible for the treatment of symptomatic uterine fibroids.

Key Points

- Magnetic-resonance-guided high intensity focused ultrasound allows non-invasive treatment of uterine fibroids.

- Volumetric feedback ablation is a novel technology that allows larger treatment volumes

- MR-guided ultrasound ablation of uterine fibroids appears safe using volumetric feedback

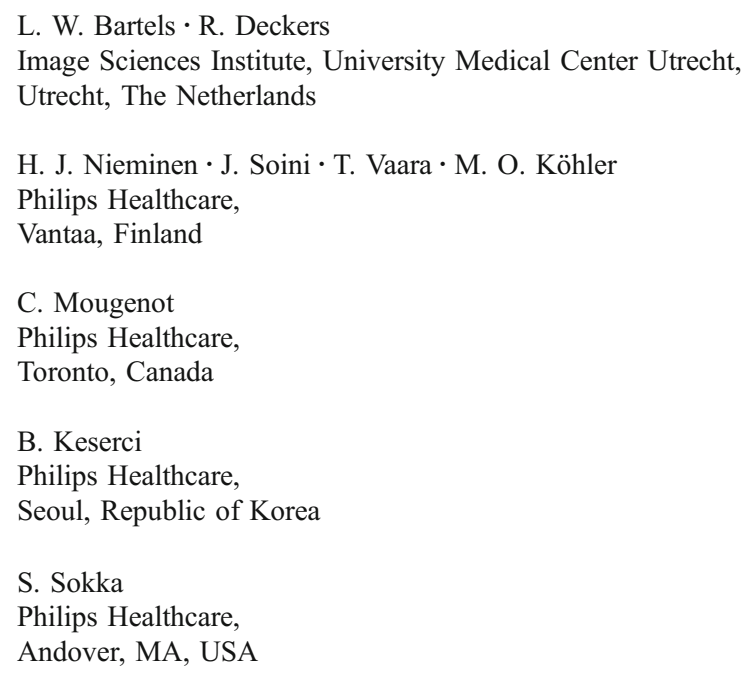


Keywords Leiomyoma $\cdot$ High intensity focused ultrasound ablation - Ultrasonic therapy - Interventional magnetic resonance imaging $\cdot$ Safety $\cdot$ Uterine fibroid

\section{Introduction}

Uterine fibroids are common benign tumors in women, with a prevalence ranging from $25 \%$ to $77 \%$ [1, 2]. Fibroids can cause menorrhagia, pelvic pain, bulk-related symptoms and infertility, resulting in a reduced quality of life. There are a variety of therapies available to achieve symptom relief, including hysterectomy, myomectomy, uterine artery embolisation and medical therapy [1-5].

Magnetic Resonance-guided High Intensity Focused Ultrasound (MR-HIFU) is a new, non-invasive treatment technique for uterine fibroids, where tissue is thermally ablated by absorption of acoustic energy delivered into the target tissue using high intensity focused ultrasound [6]. Magnetic Resonance Imaging (MRI) is used for planning and real-time monitoring of the treatment. A number of studies have shown the clinical effectiveness of MR-HIFU for fibroid treatment [7-13].

The traditional approach for MR-HIFU is performed by iterative sonication of a single focal point, with each sonication followed by a cooling period (point-by-point ablation technique) $[6,14]$. However, with this approach a relatively large portion of the delivered energy is lost via diffusion of heat out of the small targeted region, and long treatment times are required. Based on recent animal studies, a novel volumetric ablation technique of temporally switching the position of a single focal spot along outwardmoving concentric circles, has been proposed to provide a significant improvement in both treatment efficacy and ablation homogeneity [15]. Volumetric heating allows ablation of larger volumes, with diameter of treatment cells up to $16 \mathrm{~mm}$, and potentially reduces the treatment time. The purpose of this clinical trial was to assess the safety and technical feasibility of volumetric MR-HIFU ablation for treatment of patients with symptomatic uterine fibroids.

\section{Material and methods}

This multi-centre prospective study was conducted at four sites located in France, Korea, Germany, and The Netherlands. The trial (NCT00897897) was approved by the hospitals' ethical committees and country-specific regulatory bodies before trial initiation. All patients gave written informed consent for inclusion.

Included were pre- or peri-menopausal women with symptomatic uterine fibroids, 18-59 years of age, uterine size smaller than 24 weeks of pregnancy, dominant fibroid size of $\geq 3 \mathrm{~cm}$ and $\leq 12 \mathrm{~cm}$, transformed Symptom Severity Score (SSS) of $\geq 40$ points on the Uterine Fibroid Symptom and Quality of Life Questionnaire (UFS-QoL), and a normal cervical smear. Exclusion criteria were (desire for future) pregnancy, general MR imaging contraindications, other pelvic diseases, and extensive scarring of the lower abdominal wall because of the increased risk of pain or skin burns caused by such scars. Screening MR images of the pelvis were acquired in prone position (1.5 Tesla Achieva, Philips Healthcare, Best, The Netherlands) using a receiveonly torso XL coil. This included T2-weighted imaging in three orthogonal planes, and T1-weighted imaging before and after intravenous administration of a gadolinium-based contrast agent, Gadopentetate Dimeglumine (Magnevist; Schering AG, Berlin, Germany, $0.1 \mathrm{mmol}$ per $\mathrm{kg}$ body weight). MR images were used to determine the number of fibroids, location, size, treatment accessibility, contrast enhancement and presence of other pelvic diseases.

The day before treatment patients had to complete the UFS-QoL questionnaire assessing symptoms and quality of life [16] and depilated the skin of the lower abdomen. Patients arrived in the clinic in a fasting state. A Foley catheter was inserted to control bladder volume, and an intravenous catheter was used to allow administering of contrast agent and intravenous sedation with fentanyl citrate. Rectal body temperature was recorded and added

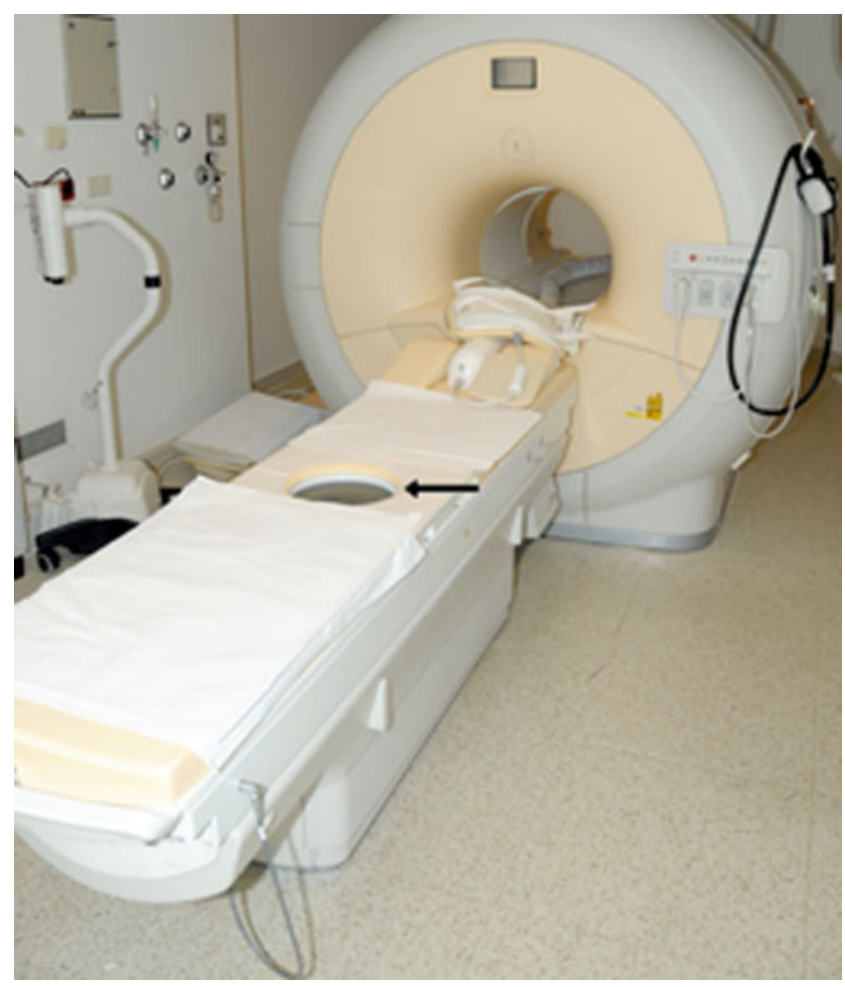

Fig. 1 The Sonalleve MR-HIFU system consists of a $1.5 \mathrm{~T}$ clinical MRI scanner and a 256-element focused ultrasound transducer integrated in the treatment table top 
Fig. 2 Schematic representation of the volumetric ablation trajectory. The electrically-steered acoustic focus is moved along concentric circular subtrajectories on a plane perpendicular to the HIFU beam producing an ellipsoidal thermal volume (diameter $=4,8,12$ or $16 \mathrm{~mm}$ )

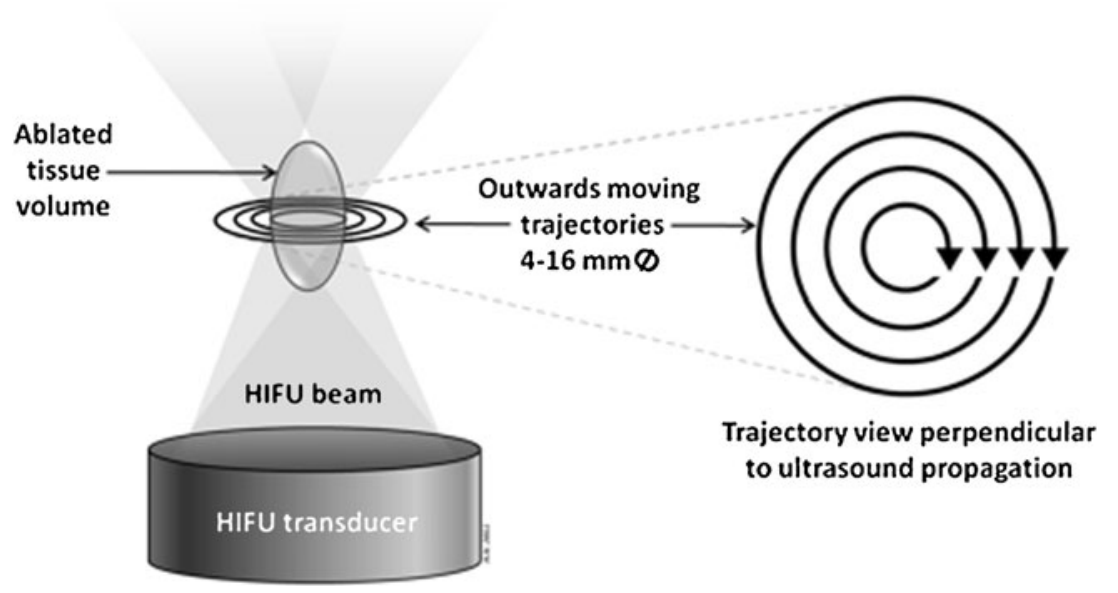

to the temperature variation maps inherent to PRF thermometry to obtain absolute temperatures as is necessary for treatment. Pain and discomfort scores were recorded before start of treatment. Pain was assessed on a scale from 0 to 10 according to the Visual Analogue Scale, where 0 stands for 'no pain' and 10 for 'worst pain imaginable' [19]. Discomfort was scored on a scale from 0 to 3: "none" (0), "mild" (1), "moderate" (2), and "severe" (3).

The patient was positioned on the HIFU tabletop in prone position, with the fibroid placed above the transducer. A gel pad and a mixture of degassed water and ultrasound gel were used to optimise acoustic coupling between the system and the patient. The patient was given a stop button to allow her to abort sonication if experiencing pain or discomfort. Treatment planning was performed based on 3D T2-weighted images of which the system automatically performed Multiplanar Reconstructions (MPRs) displaying three orthogonal views (sagittal, transversal and coronal) simultaneously to facilitate planning. Treatments were performed with the Sonalleve MR-HIFU system (Philips Healthcare, Vantaa, Finland), integrated into a 1.5 Tesla MR imaging system (Fig. 1). Ablation was performed with a volumetric technique using nominal frequencies of 1.2 or $1.45 \mathrm{MHz}$ [15]. During sonication, the transducer applied ultrasound energy in a continuous manner in a series of concentric circular trajectories of increasing size. The differently sized volumes that the user may choose to be ablated per sonication, which is also shown as a graphical object by the system, are called treatment cells. These treatment cells with nominal diameters of $4,8,12$ or $16 \mathrm{~mm}$ (and resulting treatment volumes of $0.1,0.6,2.3$, and $5.4 \mathrm{~mL}$, respectively) were planned within the target treatment area (Fig. 2). The heating produced by these volumetric sonications was measured simultaneously with sonication using Proton Resonance Frequency (PRF) shiftbased MR thermometry [17]. Temperature imaging was performed in 6 slices (3 target region coronal slices perpendicular to the beam path; 1 sagittal slice capturing the heating within the beam path; 1 near-field slice monitoring for excessive heating of the skin and fat-layer; 1 far-field slice monitoring for excessive heating at the posterior wall of the fibroid) updated every $3 \mathrm{~s}$ using gradient-echo multi-shot echo planar imaging. The system provided the option of using the online acquired temperature information for automatically controlling the sonication using a thermal feedback method [18], which stops the sonication when the measured thermal ablation and temperature profile match with that intended for the chosen treatment cell.

Immediately post-treatment, T1-weighted MR images were acquired before and after contrast administration to visualise the treatment result, i.e. the Non-Perfused Volume (NPV). Following this, the patients were taken to the recovery room and monitored before being discharged. Data on pain, discomfort, and adverse events were collected. Phone interviews addressing pain and recovery

Table 1 Patients $(n=33)$ baseline characteristics

\begin{tabular}{|c|c|}
\hline Age (years) ${ }^{\mathrm{a}}$ & $44.8 \pm 5.2$ \\
\hline Weight $(\mathrm{kg})^{\mathrm{a}}$ & $61.5 \pm 10.1$ \\
\hline Height $(\mathrm{cm})^{\mathrm{a}}$ & $163.8 \pm 6.2$ \\
\hline \multicolumn{2}{|l|}{ Race $(\%)^{b}$} \\
\hline Caucasian & $51.5(17 / 33)$ \\
\hline Asian & $30.3(10 / 33)$ \\
\hline African American & $3.0(1 / 33)$ \\
\hline South American & $6.1(2 / 33)$ \\
\hline Other & $9.1(3 / 33)$ \\
\hline \multicolumn{2}{|c|}{ Inclusions per centre $(\%)^{\mathrm{b}}$} \\
\hline France & $48.5(16 / 33)$ \\
\hline Korea & $30.3(10 / 33)$ \\
\hline Germany & $12.1(4 / 33)$ \\
\hline The Netherlands & $9.1(3 / 33)$ \\
\hline
\end{tabular}


Fig. 3 Distribution of fibroid symptoms. The average number of symptoms per patient was 3.2 out of 5

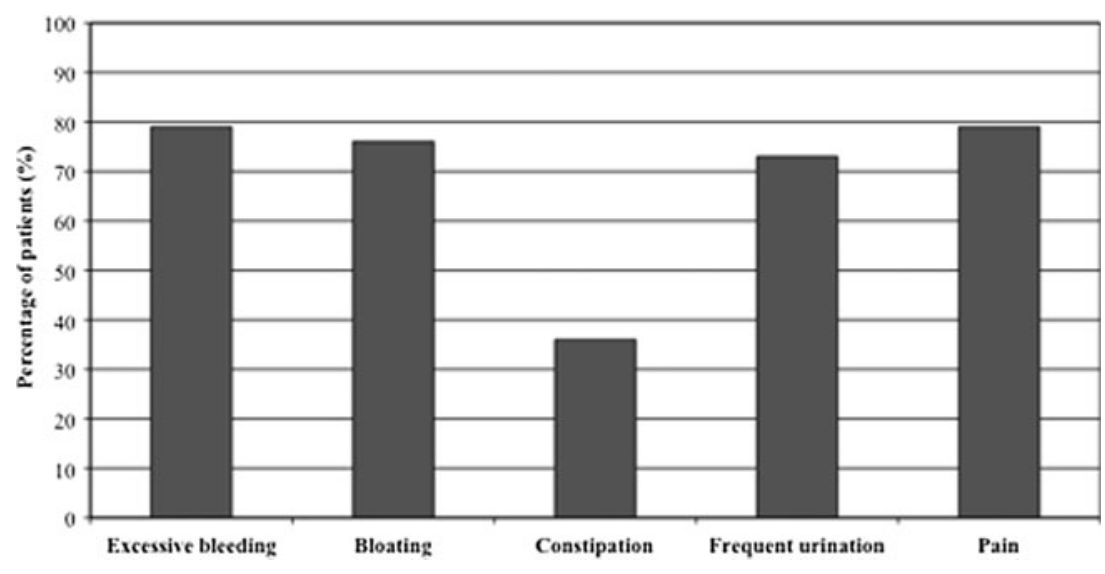

were performed 24, 48, and $72 \mathrm{~h}$ after treatment, and also at one and two weeks post-treatment. MR-imaging was scheduled 1 month after treatment. Quality of life was assessed 1 month post-treatment using the UFS-QoL [16]. Adverse Events (AEs) were recorded and classified according to the Society of Interventional Radiology (SIR) classification [20].

Technical feasibility was assessed by comparison of the NPVs with MR thermal dose predicted treatment volumes. Volume measurements of fibroids and NPVs were performed based on voxel summation. The volume of interest was manually segmented with contours on each relevant image slice. The number of voxels within each contour was calculated and the sum of voxels multiplied by the voxel volume to compute the total volume. The NPV was also calculated as a percentage of the fibroid volume (NPV ratio) [21]. Safety was determined by evaluation of complications or AEs and unintended lesions. Pain and discomfort scores, length of hospital stay, symptom improvement and quality of life, were assessed as well.

Statistical analysis was performed using the $\mathrm{SAS}^{\circledR}$ statistical package (SAS; SAS Institute, Cary, NC, USA). Descriptive statistics were used to determine the distribution of baseline data and outcome measures. Paired t-tests were used for statistical comparison between baseline and 30 days follow-up for mean pain and quality of life scores; $p$-values $<0.05$ were considered statistically significant.

\section{Results}

Thirty-three patients were enrolled in this study, with 38 treatable fibroids. Patient demographics are presented in Table 1. Three women had more than one fibroid treated. Two patients were excluded from analyses: one patient underwent uterine artery embolisation one week after MR- a

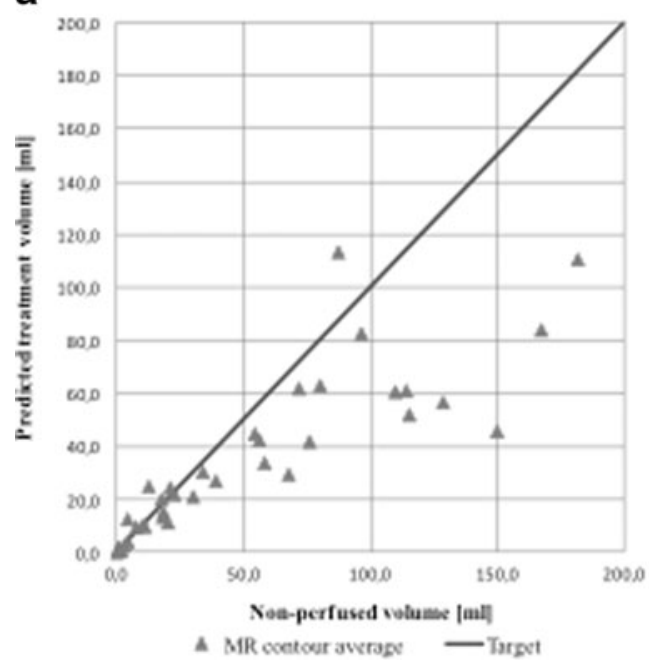

Fig. 4 a Thermal dose predicted treatment volume as a function of non-perfused volume. The diagonal line shows the target performance. b Bland-Altman plot of thermal dose predicted treatment volume and

b

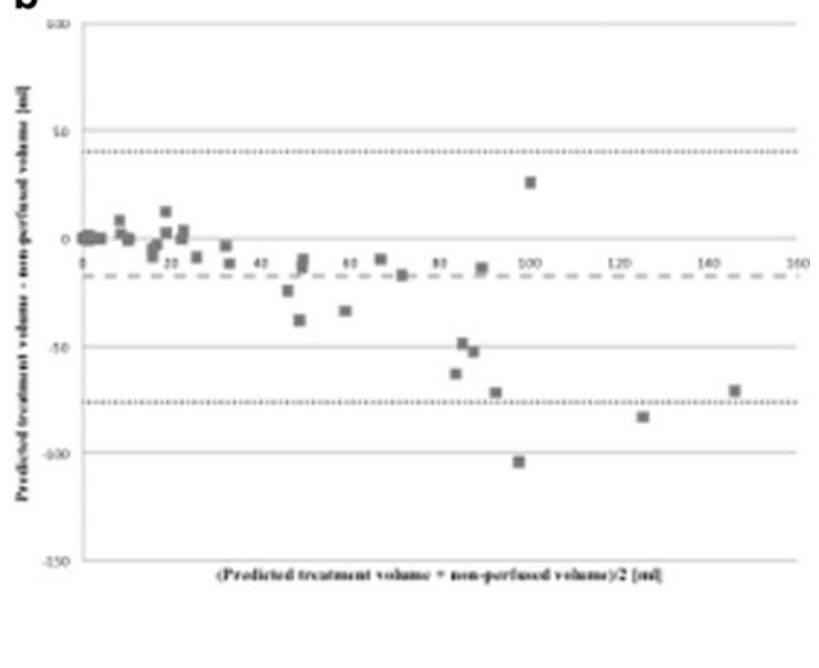

non-perfused volume showing absolute difference with mean \pm 1.96 SD acceptance limits 
Table 2 Adverse events reported in patients treated with MR-HIFU

\begin{tabular}{lc}
\hline Adverse events (AEs) & $n=31$ \\
\hline Pain & $17(55 \%)$ \\
Abdominal pain & $10(32 \%)$ \\
Positional related pain & $7(23 \%)$ \\
Sonication related pain & $1(3 \%)$ \\
Sciatic nerve pain & \\
Gynaecologic & $13(42 \%)$ \\
Abdominal discomfort & $7(23 \%)$ \\
Urinary pain or difficulty & $3(10 \%)$ \\
Vaginal bleeding post-treatment & $1(3 \%)$ \\
Haematuria & $1(3 \%)$ \\
Vaginal irritation & \\
General & $6(19 \%)$ \\
Fatigue & $4(13 \%)$ \\
Headache & $3(10 \%)$ \\
Fever $>38^{\circ}$ Celsius & $3(10 \%)$ \\
Nausea & $3(10 \%)$ \\
Skin irritation & $6(19 \%)$ \\
Other & 85 \\
Total &
\end{tabular}

Data are number of patients, with percentages in parentheses

One patient may have experienced more than one $\mathrm{AE}$

HIFU treatment, because of unsatisfactory treatment results due to insufficient heating of the fibroid, and the other patient underwent surgical fibroid removal in another hospital for unknown reasons. Figure 3 provides an overview of the fibroid symptoms in the 31 patients that were finally included, the most commonly reported symptoms being excessive menstrual bleeding and pain.

Technical feasibility was assessed by comparison of the actual MR-measured NPVs with predicted treatment volumes based on MR thermal dose maps. The mean NPV calculated as a percentage of the total fibroid volume was $21.7 \%$ (range $0-66 \%$ ), whereas the mean predicted treatment volume was $34.5 \mathrm{~mL} \pm 30.1$. Figure $4 \mathrm{a}$ displays the predicted treatment volume as a function of the NPV for

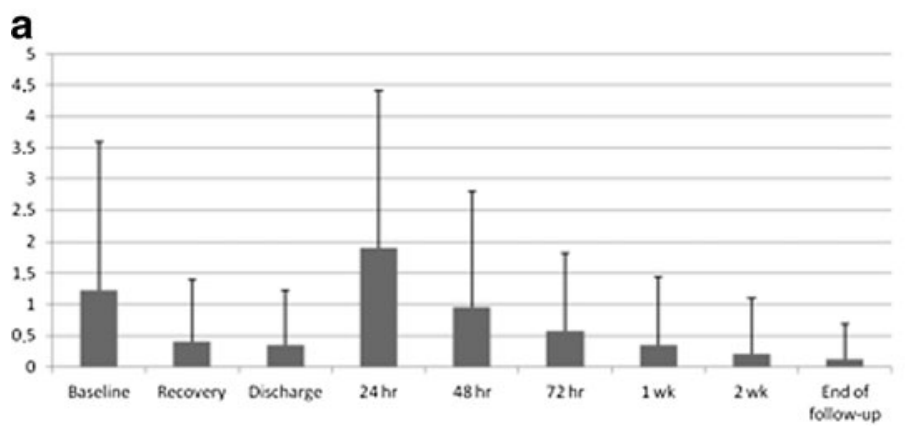

Fig. 5 Mean pain (a, range 0-10) and discomfort (b, range 0-3) scores (including error bars) at consecutive follow-up points

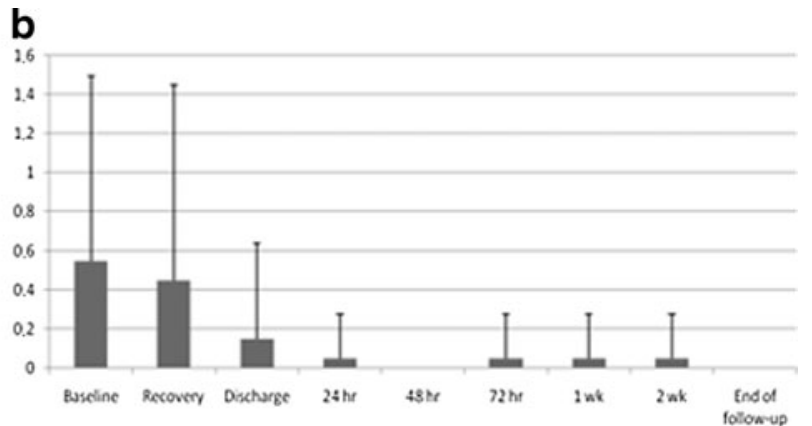

each fibroid. The correlation coefficient $r$ was 0.87 , representing a very strong correlation. Figure $4 \mathrm{~b}$ displays the corresponding Bland-Altman plot of thermal dose predicted treatment volume and NPV showing the absolute difference with mean $\pm 1.96 \mathrm{SD}$ acceptance limits.

No serious adverse events occurred. A total of 85 AEs were reported in 31 patients, with a mean of 2.6 AEs per patient (Table 2). None of the AEs were major according to the SIR classification [20]. AEs typically resolved within 3 days post-treatment, however two patients reported ongoing AEs at 30 days follow-up (one patient with sciatic nerve pain, spontaneously resolved after 139 days, and one patient with leg pain, resolved after 93 days post-treatment). Figure 5 shows pain and discomfort scores during followup. Mean baseline reported pain score was $1.8 \pm 2.6$, with an increase in pain score to $2.4 \pm 2.6,24 \mathrm{~h}$ post-treatment, however this increase was not significant $(p=0.343)$. At the end of follow-up, the mean pain score was significantly reduced to $0.1 \pm 0.5(p=0.002)$. Mean baseline discomfort was $0.8 \pm 0.8$, decreasing to $0.18 \pm 0.50$ at hospital discharge. Mean length of hospital stay was $9.3 \mathrm{~h} \pm 3.3$, with a maximum of $17.0 \mathrm{~h}$. Time needed to return to work and normal activities ranged from 1-19 days (mean 4.4 14.4 ) and $1-7$ days (mean $2.3 \pm 1.8$ ) respectively.

\section{Discussion}

Our results showed that volumetric ablation of uterine fibroids with the Sonalleve MR-HIFU system is both safe and technically feasible. No serious adverse events occurred, and concordance between the predicted treatment volume and the NPV was found in 33 out of 36 treated fibroids (concordance rate of $92 \%$ ).

In this study we used the volumetric MR-HIFU Sonalleve system for treatment of patients with symptomatic fibroids. Previous studies used the ExAblate 2000 (InSightec, Haifa, Israel) HIFU system, which uses a different ablation strategy [6]. The main advantage of the volumetric ablation technique is that it allows for a 
controlled heating of a larger volume per sonication by rapid spiral wise movement of the focal spot. Additionally thermal ablation is controlled utilising feedback control [15]. This could potentially reduce treatment time for a given treatment volume in future studies. The aim of this feasibility study was to assess safety and technical feasibility. The technical performance rate we found for the Sonalleve system is at least equal to those reported in the literature for the ExAblate 2000. In our study, the ratio between NPV and the predicted treatment volume is $1.4 \pm 0.6$, while McDannold reported a ratio of 1.9 for the ExAblate 2000 system between the non-perfused area and the predicted treatment area in the central coronal plane of the treatment [21]. The match between treated volumes (NPVs) and predicted treatment volumes in our study was very good with a $92 \%$ concordance rate that may in part be attributed to the homogeneous ablation with sharp thermal dose borders produced by the utilised volumetric ablation technique. The findings of McDannold are also supported by two earlier feasibility studies from Stewart et al. and Tempany et al. that showed similar results $[6,14,21,23]$.

Also the safety profile of the Sonalleve system is similar to what has been reported in the literature for the ExAblate 2000. Minor adverse events were reported such as abdominal tenderness, nausea, or first-degree skin burns. Treatments were performed on an outpatient basis $[6,14,22]$. Since this feasibility study focused on safety of a novel MR-HIFU system for treatment of patients with uterine fibroids, a potential limitation is its small population included in the study and the relatively short reported patient follow-up of one month. Longer follow-up will be needed to report the clinical efficacy of the treatment as quantified by improvement of symptoms and an increased quality of life already of patients treated. These outcomes will be reported in a separate article. Another limitation of this study was that only a maximum of $50 \%$ ablation of the fibroid volume was allowed. Recent studies have shown that treatment success is largely dependent on the ablation volume and the percentage of ablated fibroid tissue, with increased fibroid shrinkage, improved symptom relief, and fewer additional treatments being obtained when larger volumes of the fibroid are ablated [7, 8, 24, 25]. Fibroid shrinkage is especially relevant for women suffering from bulk-related symptoms. MR-HIFU treatment should therefore be focused on treating as much fibroid tissue as possible. Reducing safety margins to sensitive structures such as the bowel and the uterine serosa is inevitable to achieve this, and has already proven to be safe [26, 27].

In conclusion, this study proved that volumetric MRHIFU with the Sonalleve system is safe and technically feasible for the treatment of symptomatic uterine fibroids. Future studies will be aimed at treatment of more patients with larger ablation volumes. Longer follow-up periods will be required to provide information about clinical outcome.

Open Access This article is distributed under the terms of the Creative Commons Attribution Noncommercial License which permits any noncommercial use, distribution, and reproduction in any medium, provided the original author(s) and source are credited.

\section{References}

1. Buttram VC Jr, Reiter RC (1981) Uterine leiomyomata: etiology, symptomatology, and management. Fertil Steril 36:433-445

2. Stewart EA (2001) Uterine fibroids. Lancet 357:293-298

3. Williams VS, Jones G, Mauskopf J et al (2006) Uterine fibroids: a review of health-related quality of life assessment. J Womens Health (Larchmt) 15:818-829

4. Bradley LD (2009) Uterine fibroid embolization: a viable alternative to hysterectomy. Am J Obstet Gynecol 201:127135

5. Goodwin SC, Spies JB, Worthington-Kirsch R et al (2008) Uterine artery embolization for treatment of leiomyomata: longterm outcomes from the FIBROID Registry. Obstet Gynecol 111:22-33

6. Tempany CM, Stewart EA, McDannold N et al (2003) MR imaging-guided focused ultrasound surgery of uterine leiomyomas: a feasibility study. Radiology 226:897-905

7. LeBlang SD, Hoctor K, Steinberg FL (2010) Leiomyoma shrinkage after MRI-guided focused ultrasound treatment: report of 80 patients. AJR Am J Roentgenol 194:274-280

8. Fennessy FM, Tempany CM, McDannold NJ et al (2007) Uterine leiomyomas: MR imaging-guided focused ultrasound surgery-results of different treatment protocols. Radiology 243:885-893

9. Funaki K, Fukunishi H, Sawada K (2009) Clinical outcomes of magnetic resonance-guided focused ultrasound surgery for uterine myomas: 24-month follow-up. Ultrasound Obstet Gynecol 34:584-589

10. Stewart EA, Gostout B, Rabinovici J et al (2007) Sustained relief of leiomyoma symptoms by using focused ultrasound surgery. Obstet Gynecol 110:279-287

11. Hesley GK, Felmlee JP, Gebhart JB et al (2006) Noninvasive treatment of uterine fibroids: early Mayo Clinic experience with magnetic resonance imaging-guided focused ultrasound. Mayo Clin Proc 81:936-942

12. Carls GS, Lee DW, Ozminkowski RJ et al (2008) What are the total costs of surgical treatment for uterine fibroids? J Womens Health (Larchmt ) 17:1119-1132

13. Zowall H, Cairns JA, Brewer C et al (2008) Cost-effectiveness of magnetic resonance-guided focused ultrasound surgery for treatment of uterine fibroids. BJOG 115:653-662

14. Stewart EA, Gedroyc WM, Tempany CM et al (2003) Focused ultrasound treatment of uterine fibroid tumors: safety and feasibility of a noninvasive thermoablative technique. Am J Obstet Gynecol 189:48-54

15. Köhler MO, Mougenot C, Quesson B et al (2009) Volumetric HIFU ablation under 3D guidance of rapid MRI thermometry. Med Phys 36:3521-3535

16. Chapman A, ter Haar G (2007) Thermal ablation of uterine fibroids using MR-guided focused ultrasound-a truly non-invasive treatment modality. Eur Radiol 17:2505-2511 
17. Ishihara Y, Calderon A, Watanabe $H$ et al (1995) A precise and fast temperature mapping using water proton chemical shift. Magn Reson Med 34:814-823

18. Enholm JK, Köhler MO, Quesson B et al (2010) Improved volumetric MR-HIFU ablation by robust binary feedback control. IEEE Trans Biomed Eng 57:103-113

19. Huskisson EC (1974) Measurement of pain. Lancet 2:11271131

20. Hovsepian DM, Siskin GP, Bonn J et al (2004) Quality improvement guidelines for uterine artery embolization for symptomatic leiomyomata. Cardiovasc Intervent Radiol 27:307-313

21. McDannold N, Tempany CM, Fennessy FM et al (2006) Uterine leiomyomas: MR imaging-based thermometry and thermal dosimetry during focused ultrasound thermal ablation. Radiology 240:263-272

22. Hindley J, Gedroyc WM, Regan L et al (2004) MRI guidance of focused ultrasound therapy of uterine fibroids: early results. AJR Am J Roentgenol 183:1713-1719
23. Hynynen K, Colucci V, Chung A et al (1996) Noninvasive arterial occlusion using MRI-guided focused ultrasound. Ultrasound Med Biol 22:1071-1077

24. Lenard ZM, McDannold NJ, Fennessy FM et al (2008) Uterine leiomyomas: MR imaging-guided focused ultrasound surgery-imaging predictors of success. Radiology 249:187194

25. Kroencke TJ, Scheurig C, Poellinger A et al (2010) Uterine artery embolization for leiomyomas: percentage of infarction predicts clinical outcome. Radiology 255:834-841

26. Morita Y, Ito N, Hikida $\mathrm{H}$ et al (2008) Non-invasive magnetic resonance imaging-guided focused ultrasound treatment for uterine fibroids - early experience. Eur J Obstet Gynecol Reprod Biol 139:199-203

27. Zhang L, Chen WZ, Liu YJ et al (2010) Feasibility of magnetic resonance imaging-guided high intensity focused ultrasound therapy for ablating uterine fibroids in patients with bowel lies anterior to uterus. Eur J Radiol 73:396-403 